\title{
Efficacy of magnetic resonance imaging with an SPGR sequence for the early evaluation of knee cartilage degeneration and the relationship between cartilage and other tissues
}

Xin Yang ${ }^{1+}$, Zhuoyang $\mathrm{Li}^{2+}$, Yongping Cao ${ }^{1 *}$, Yufeng $\mathrm{Xu}^{3}$, He Wang ${ }^{3}$, Licheng Wen ${ }^{1}$, Zhichao Meng ${ }^{1}$, Heng Liu', Rui Wang ${ }^{1}$ and Xiang Li ${ }^{1}$

\begin{abstract}
Rationale and objectives: The aim of this study was to investigate the efficacy of magnetic resonance imaging (MRI) with a spoiled gradient-recalled (SPGR) sequence to evaluate early knee cartilage degeneration and the relationship between cartilage and other tissues using a modified Whole-Organ Magnetic Resonance Imaging Score (WORMS).

Materials and methods: Eighty-four patients with knee joint pain were evaluated by X-ray and MRI with an SPGR sequence from June 2015 to December 2016. Joint degeneration was graded by two experienced radiologists using the Kellgren-Lawrence (K-L) grading scale. The modified WORMS was used to evaluate cartilage lesions, bone marrow abnormalities, bone cysts, osteophytes, joint effusion and synovitis. The difference between the WORMS of the SPGR and the T2 sequences evaluated by the Wilcoxon signed-rank test was determined, and the relationships between the WORMS features were evaluated by a Spearman correlation.

Results: The modified WORMS for the cartilage lesion evaluation was significantly higher with the SPGR sequence than with the $\mathrm{T} 2$ sequence $(P<0.05)$. The cartilage lesions showed a moderate correlation with osteophytes, synovitis and joint effusion (Rs $>0.40, P<0.05$ ) and weak correlations with bone marrow abnormalities and bone cysts ( $\operatorname{Rs}<0.4, P<0.05$ ).

Conclusion: The modified WORMS evaluation using MRI with the SPGR sequence was much better than the normal sequence for early knee osteoarthritis (OA). The cartilage lesions are associated with bone marrow abnormalities and the other features of $O A$.
\end{abstract}

Keywords: Osteoarthritis, Magnetic resonance imaging, Spoiled gradient-recalled sequence, Cartilage lesions

\section{Introduction}

Magnetic resonance imaging (MRI) is a common noninvasive imaging diagnostic technique for soft tissue. However, cartilage lesions cannot be distinctly observed via the typically used MRI sequences [1]. The three-dimensional fat-suppressed spoiled gradient-recalled (3D-FS-SPGR)

\footnotetext{
* Correspondence: freehorse6666@163.com

${ }^{+}$Xin Yang and Zhuoyang Li contributed equally to this study and share first authorship.

${ }^{1}$ Department of Orthopedics, Peking University First Hospital, No. 8, Xishiku

Street, Beijing 100034, Beijing, China

Full list of author information is available at the end of the article
}

sequence, which has a high contrast/noise ratio (CNR), can improve the contrast between the cartilage and the surrounding bone marrow by fat suppression to better display the cartilage [2]. There are few studies that report the usage of the SPGR sequence for the Whole-Organ Magnetic Resonance Imaging Score (WORMS). The purpose of this paper is to investigate the diagnostic accuracy of a modified WORMS using MRI with the SPGR sequence for the evaluation of early knee osteoarthritis (OA).

(c) The Author(s). 2019 Open Access This article is distributed under the terms of the Creative Commons Attribution 4.0 International License (http://creativecommons.org/licenses/by/4.0/), which permits unrestricted use, distribution, and 


\section{Materials and methods Subjects}

MRI with the SPGR sequence and radiographs from 84 patients were collected between June 2015 and December 2016. This study was approved by the Ethics Committee of our hospital. The REB number was (2014[713]). The patients who had chronic mild knee pain and met the American College of Rheumatology guidelines for knee OA [3] were included. The inclusion criteria were as follows: (1) patients had mild knee pain with Visual Analogue Scale (VAS) scores between 2 and $4 \mathrm{~cm}$ [4]; (2) at least 3 months of pain duration [5], and (3) the severity of radiographic OA was between grade 0 and 3 graded with the Kellgren-Lawrence (K-L) grading scale [6]. The exclusion criteria were as follows: (1) known diagnosis of inflammatory or metabolic diseases, such as rheumatoid arthritis, gout, tuberculosis or septic arthritis; (2) neoplastic diseases of the examined knee; (3) intra-articular bone fracture and ligament rupture of the knee joint; and (4) recent treatment prior to the imaging study. The mean age was 48.9 years (17-75 years). Thirty-four patients $(40.48 \%)$ were men, and 50 patients $(59.52 \%)$ were women. The left knee was involved in 37 patients (44.05\%), and the right knee was involved in 47 patients (55.95\%).

\section{MRI parameters}

The patients were told to assume a supine position, and the knee joints were held in the centre of the coil to maintain their position during the examination. We used a 3.0 Tesla whole-body GE MR750 scanner (General Electric Healthcare, Milwaukee, WI) with an 8-channel knee coil. The imaging sequences included Cor-PDw (SE 2500/35 [TR msec/TE msec], 18-cm field of view [FOV], $4 \mathrm{~mm} / 1 \mathrm{~mm}$ [slice thickness/interslice gap], $320 \times 256$ matrix, echo train length [ETL] of 10), Sag-T1w (655/12, $18-\mathrm{cm}$ FOV, $4 \mathrm{~mm} / 0.4 \mathrm{~mm}, 384 \times 256,3), \quad$ Ax-T $2 \mathrm{w}$ (2500/42, 18-cm FOV, $4 \mathrm{~mm} / 1 \mathrm{~mm}, 384 \times 256$, 12), sagittal PD/T2w dual echo (2500/30/85, 18-cm FOV, $4 \mathrm{~mm} /$ $0.4 \mathrm{~mm}, 352 \times 256,12)$ and 3D-FS-SPGR $(7.5 / 2.9,18-\mathrm{cm}$ FOV, $2 \mathrm{~mm} / 0 \mathrm{~mm}, 256 \times 256$ matrix, $15^{\circ}$ flip angle).

\section{Data analysis}

After two hours of training for performing the WORMS, two experienced registered radiologists who had 10 years of work experience independently evaluated the radiographs and MRI data.

\section{Radiographs}

The severity of radiographic OA was graded via the K-L grading scale as follows: $0=$ normal; $1=$ possible joint space narrowing and osteophyte formation; 2 =definite osteophyte formation with possible joint space narrowing; 3 = multiple osteophytes, definite joint space narrowing, sclerosis and possible bony deformity; and $4=$ large
Table 1 The K-L grading scale distribution for the patients

\begin{tabular}{lllll}
\hline K-L grading & 0 & 1 & 2 & 3 \\
\hline Total & 0 & 36 & 29 & 19
\end{tabular}

K-L Kellgren-Lawrence

osteophytes, marked joint space narrowing, severe sclerosis and definite bony deformity [6]. Each radiologist read the radiographs randomly to confirm the $\mathrm{K}-\mathrm{L}$ grade. If there was a different decision from the two radiologists, the K-L grade was confirmed by our orthopaedic professor who had 30 years of work experience.

\section{MRI evaluation}

The joint degeneration in knee OA was graded with the WORMS [7]. The knee joint was divided into four major regions: medial and lateral tibiofemoral joints, the patellofemoral joint and the portion of the tibia beneath the tibial spines (subspinous). Six features were evaluated using the WORMS, including the cartilage lesion, bone marrow abnormalities, bone cysts, osteophytes, synovitis and joint effusion. Cartilage lesions were graded in the four regions using the Recht scale [8]: $0=$ normal, $1=$ areas of inhomogeneous signal intensity of the articular cartilage on the sequences, 2 = defects involving less than half of the articular cartilage thickness, 3 = defects involving more than half of the cartilage but less than the full thickness and 4 = full-thickness defects exposing the bone. Synovitis was graded using the estimated maximal distention of the synovial cavity: $0=$ normal, $1=<33 \%$ of maximum potential distention, $2=33-66 \%$ of maximum potential distention and $3=>66 \%$ of maximum potential distention [7].

Bone marrow abnormalities and bone cysts were graded on a 4 -point scale: $0=$ normal, $1=<25 \%$ of the region, 2 $=25$ to $50 \%$ of the region and $3=>50 \%$ of the region [7]. Osteophytes and joint effusion were graded as follows: 0 = none, $1=$ mild, $2=$ moderate and $3=$ severe [7].

\section{Statistical analysis}

Statistical analysis was performed using the SPSS 23.0 software package (Chicago, IL, USA). Fleiss $\kappa$ was used to compare inter-rater agreement for determining the $\mathrm{K}-\mathrm{L}$ grade of the radiographic OA. The $95 \%$ confidence intervals for

Table 2 The frequencies of the modified WORMS features in the different joint regions as determined with the SPGR sequence

\begin{tabular}{|c|c|c|c|c|}
\hline \multirow[t]{2}{*}{ WORMS feature } & \multicolumn{4}{|l|}{ Regions } \\
\hline & $\overline{\mathrm{MFTJ}}$ & LFTJ & PFJ & $\mathrm{S}$ \\
\hline Cartilage lesions & $29(34.52 \%)$ & $15(17.86 \%)$ & $41(48.81 \%)$ & \\
\hline $\mathrm{BMA}$ & $26(30.95 \%)$ & 19 (22.62\%) & 33 (39.29\%) & $8(0.95 \%)$ \\
\hline Bone cysts & $7(8.33 \%)$ & $5(5.95 \%)$ & $8(9.52 \%)$ & $5(5.95 \%)$ \\
\hline
\end{tabular}

WORMS Whole-Organ Magnetic Resonance Imaging Score, SPGR spoiled gradient-recalled, MFTJ Medial tibiofemoral joint, LFTJ lateral tibiofemoral joint, PFJ patellofemoral joint, $S$ subspinous, $B M A$ bone marrow abnormalities 
the $\kappa$ values were calculated for the inter-observer reliability. The frequencies of the modified WORMS features, such as cartilage lesions, bone marrow abnormalities and bone cysts, were calculated when a patient had a score over 0 for different joint regions. The cartilage lesion scores and the WORMS were evaluated by the SPGR sequence and T2 sequence in different K-L grades and were analysed by the Wilcoxon signed-rank test. The difference was considered significant at $P<0.05$. Spearman correlations were used to quantify the associations between the K-L grade and the sum of the WORMS in the primary analysis.

For the secondary analysis, the associations between cartilage lesions and other WORMS abnormalities were examined, and the sum of the cartilage lesion scores and other features were calculated for each region and compared amongst regions.

Spearman's rho (Rs) was used to describe the Spearman correlations. We defined a 3-point scale as follows: $0-0.4=$ weak correlation, $0.4-0.7=$ moderate correlation and $>0.7=$ significant correlation [9].

\section{Results}

\section{X-ray and MRI findings}

The distribution of the K-L grading was as follows: grade 1, 36 cases; grade 2, 29 cases; and grade 3, 19 cases (Table 1). The Fleiss $\kappa$ value was 0.77 (95\% confidence interval, 0.73 to 0.82 ), indicating substantial agreement. According to the results of the MRI with the SPGR sequence, cartilage lesions, bone marrow abnormalities and bone cysts were approximately equally distributed amongst the different regions of the joint (Table 2). The highest frequency of cartilage lesions was in the patellofemoral joint (41 knees, 48.81\%), while the lowest frequency was in the lateral tibiofemoral joint (15 knees, $17.86 \%)$. The subchondral bone marrow abnormalities were mostly present in the patellofemoral joint (33 knees, 39.29\%) and rarely in the subspinous (8 knees, $0.95 \%)$. The results for the bone cysts showed that the patellofemoral joint had the highest frequency $(8 \mathrm{knees}$, $9.52 \%)$, whereas both the lateral tibiofemoral joint and the subspinous had the lowest frequency (5 knees, $5.95 \%)$. The mean scores for the WORMS in the SPGR sequence are shown in Fig. 1. The mean score for cartilage lesions, subchondral bone marrow abnormalities, bone cysts, osteophytes, joint effusion and synovitis were $3.85 \pm 4.72, \quad 2.43 \pm 3.43, \quad 0.48 \pm 1.06, \quad 0.83 \pm 0.77, \quad 1.19 \pm$ 0.48 and $0.73 \pm 0.88$, respectively.

\section{Comparison of cartilage scores for SPGR and T2 sequences}

According to the findings for the MRI with the SPGR sequence, five layers with different signal intensities were visible on the normal cartilage of the patella or femoral condyle (Fig. 2a, b). For grade 1 cartilage lesions, the low signal intensity in the cartilage and the high signal intensity in the subchondral bone appeared distinctly in the SPGR sequence (Fig. 2c). These represented a cartilage lesion and bone marrow

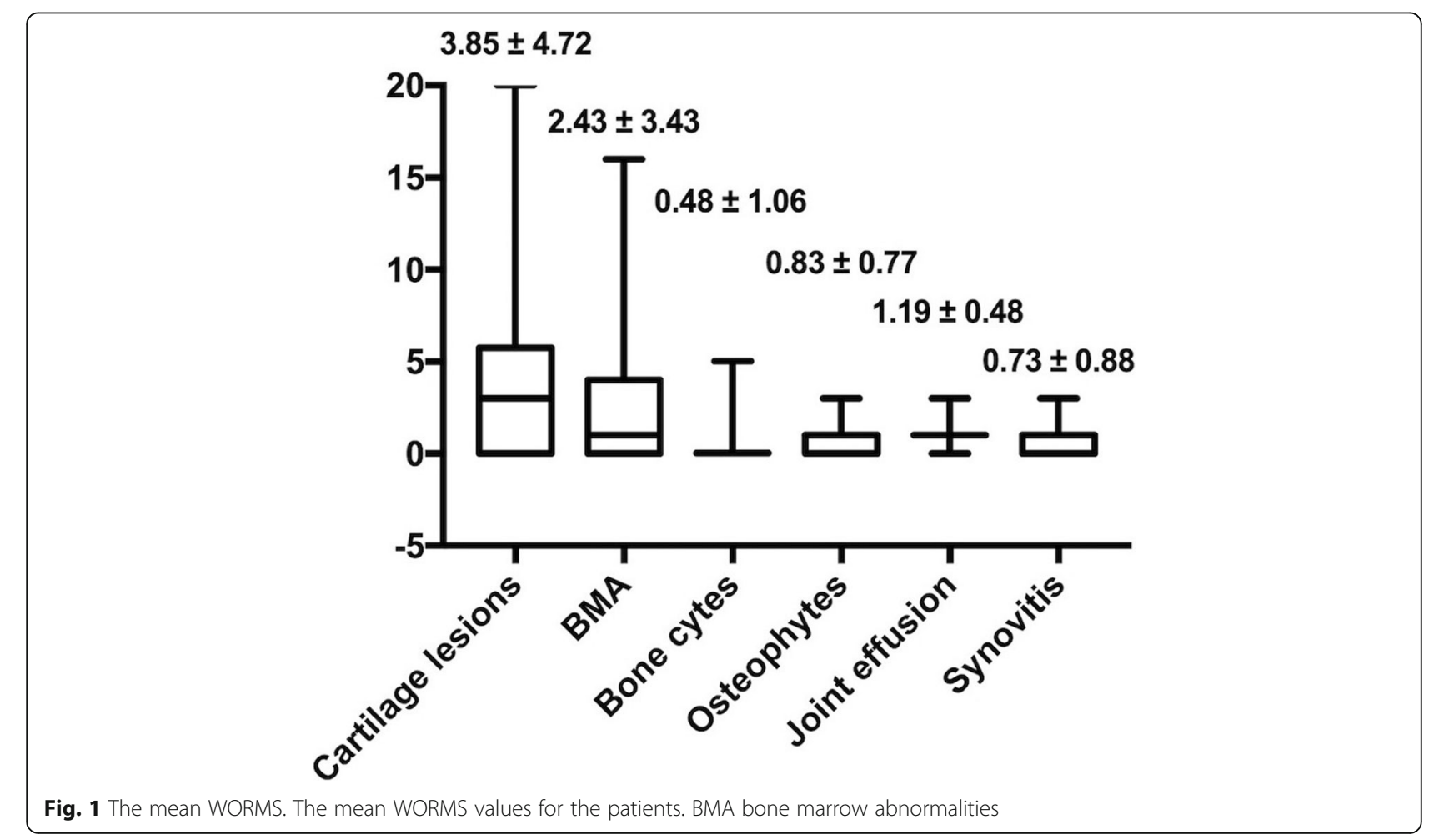




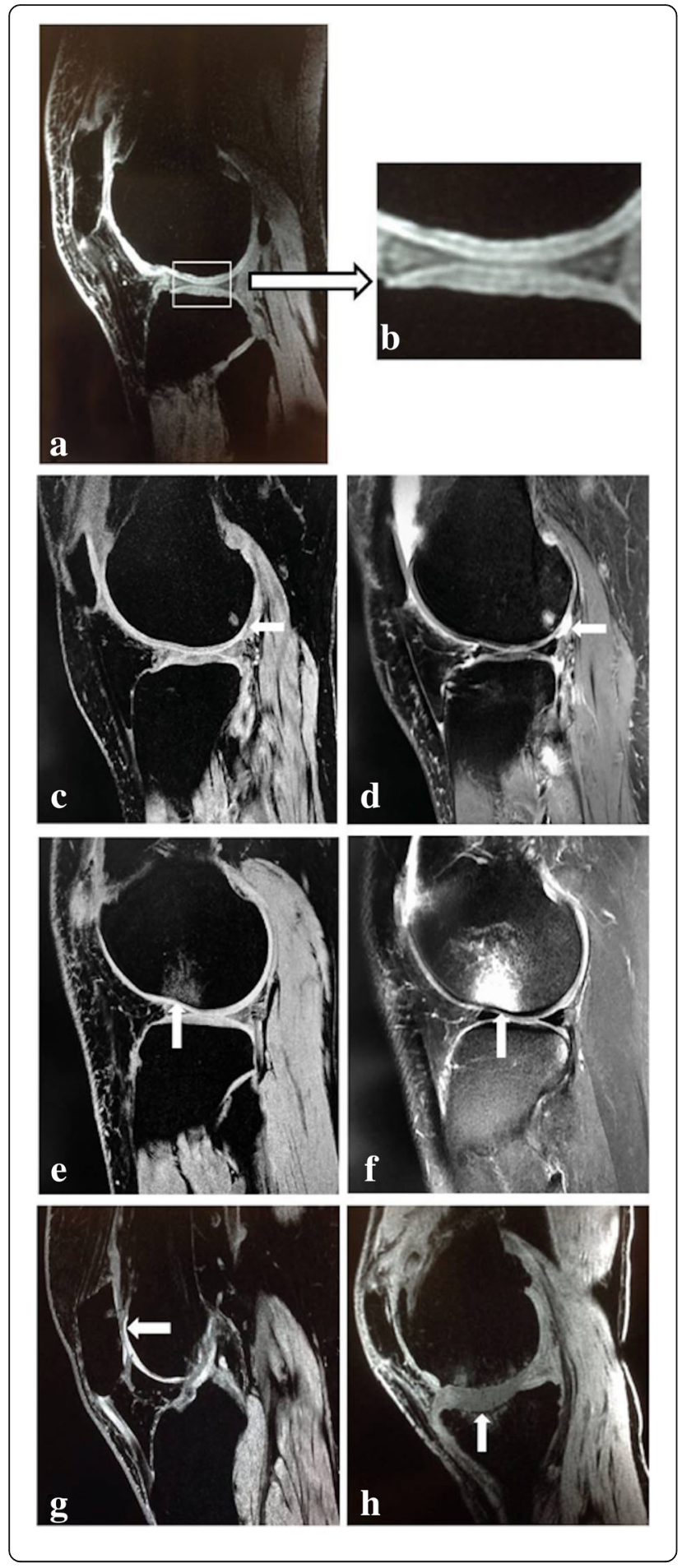

Fig. 2 Normal cartilage and different grade lesions for the SPGR and T2 sequences. $\mathbf{a}$ and $\mathbf{b}$ show the five-layered cartilage structure of the tibial plateau and the three-layered cartilage structure of the femoral condyle. For the grade 1 cartilage lesion (arrow) in c, a low signal intensity for the cartilage distinctly appeared. The cartilage lesion (arrow) in $\mathbf{d}$ was covered by joint effusion, and the border between the cartilage and the joint effusion could not be

distinguished with the T2 sequence. The articular cartilage layer disappeared, and the defect was less than $50 \%$ of the thickness of normal cartilage for a grade 2 cartilage lesion (arrow in e). The T2 sequence shown in $\mathbf{f}$ also shows a mixed signal for a cartilage lesion (arrow) and joint effusion. $\mathbf{g}$ shows a grade 3 patellar cartilage lesion (arrow) with a defect of up to $50 \%$ of the thickness of normal cartilage. $\mathbf{h}$ shows severe wear of the cartilage of the femur, tibia and patella, which is considered to be a grade 4 cartilage lesion, and the subchondral bone marrow exhibits a cystic or patchy high signal

abnormalities. However, in the T2 sequence, the cartilage lesion was covered by joint effusion, and the border between the cartilage and the joint effusion could not be distinguished (Fig. 2d). For the grade 2 lesions, the articular cartilage layer disappeared, the shape was irregular, and the defect was less than 50\% of the normal cartilage thickness in the SPGR sequence (Fig. 2e). The T2 sequence also showed a mixed signal for cartilage lesions and joint effusion (Fig. 2f). Figure 2g shows the complete cartilage contours of the lateral femoral condyle, no abnormal signals in the cartilage, and a clear three-layer structure. However, the patellar cartilage showed grade 3 lesions, and the defect was up to $50 \%$ of the normal cartilage thickness in the SPGR sequence. A grade 4 cartilage lesion is shown in Fig. 2h. The cartilage of the femur, tibia, and patella was worn, and the subchondral bone marrow showed a cystic or patchy high signal.

The frequency and mean score for the cartilage lesions evaluated by MRI with the SPGR and T2 sequences are shown in Table 3. As the K-L grade of the X-rays increased, the frequency of cartilage lesions according to both SPGR and T2 sequences increased. In the K-L1 grade, the frequency evaluated by MRI with the SPGR sequence was $38.89 \%$, and it was $19.44 \%$ when evaluated by MRI with the T2 sequence. The mean score for the cartilage lesion evaluation with the SPGR sequence was $1.19 \pm 1.92$, which was higher than that with the T2 sequence $(0.39 \pm 0.96)(P<0.05)$ (Table 3$)$. For the K-L2 grade, the frequencies of cartilage lesions were $72.41 \%$ and $58.62 \%$ using MRI with the SPGR and T2 sequences, respectively. The mean scores obtained with the SPGR and T2 sequences were $3.21 \pm 2.79$ and $1.76 \pm$ 2.50 , respectively; this difference was significant $(P<$ 0.05 ) (Table 3). The frequency of cartilage lesions was the same when using the SPGR and T2 sequences in the K-L3 grade (89.47\%); however, the score with the SPGR 
Table 3 Comparison of frequencies of cartilage lesions, the cartilage lesion scores and the modified WORMS for the SPGR and T2 sequences for the different K-L grades

\begin{tabular}{|c|c|c|c|c|}
\hline \multirow[t]{2}{*}{ K-L grading scale } & & & \multicolumn{2}{|l|}{ MRI sequence } \\
\hline & & & $\overline{\text { SPGR }}$ & T2 \\
\hline \multirow[t]{3}{*}{$\overline{\mathrm{K}-\mathrm{L} 1 \text { grade }}$} & Cartilage lesion & Frequency & $14 / 36$ (38.89\%) & $7 / 36(19.44 \%)$ \\
\hline & & Mean \pm SD & $1.19 \pm 1.92^{*}$ & $0.39 \pm 0.96^{*}$ \\
\hline & WORMS & & $4.78 \pm 4.31^{*}$ & $4.06 \pm 3.79^{*}$ \\
\hline \multirow[t]{3}{*}{ K-L2 grade } & Cartilage lesion & Frequency & $21 / 29$ (72.41\%) & $17 / 29(58.62 \%)$ \\
\hline & & Mean \pm SD & $3.21 \pm 2.79^{*}$ & $1.76 \pm 2.50^{*}$ \\
\hline & WORMS & & $8.38 \pm 4.95^{*}$ & $7.00 \pm 5.00^{*}$ \\
\hline \multirow[t]{3}{*}{ K-L3 grade } & Cartilage lesion & Frequency & $17 / 19$ (89.47\%) & 17/19 (89.47\%) \\
\hline & & Mean $\pm S D$ & $9.84 \pm 5.55^{*}$ & $6.84 \pm 5.04^{*}$ \\
\hline & WORMS & & $20.16 \pm 7.90^{*}$ & $17.16 \pm 7.59^{*}$ \\
\hline \multirow[t]{3}{*}{ Total } & Cartilage lesion & Frequency & $52 / 84(61.90 \%)$ & $41 / 84(48.80 \%)$ \\
\hline & & Mean \pm SD & $3.85 \pm 4.72^{*}$ & $2.38 \pm 3.86^{*}$ \\
\hline & WORMS & & $9.50 \pm 8.11^{*}$ & $8.04 \pm 7.30^{*}$ \\
\hline
\end{tabular}

sequence was higher than that with the T2 sequence $(9.84 \pm 5.55$ vs. $6.84 \pm 5.04) \quad(P<0.05) \quad$ (Table 3$)$. The WORMS with the SPGR sequence for the K-L1, K-L2 and K-L3 grades were $4.78 \pm 4.31,8.38 \pm 4.95$ and 20.16 \pm 7.90 , respectively; with the $\mathrm{T} 2$ sequence, whereas with the WORMS, they were $4.06 \pm 3.79,7.00 \pm 5.00$ and $17.16 \pm 7.59$, respectively; and the differences in all grades were significant $(P<0.05)$ (Table 3$)$.

The mean score for the cartilage lesion evaluation with the SPGR sequence was $3.85 \pm 4.72$, which was higher than that with the T2 sequence $(2.38 \pm 3.86)$; this difference was significant $(P<0.05)$ (Table 3$)$. The WORMS with the SPGR sequence was $9.50 \pm 8.11$, which was also higher than that with the T2 sequence $(8.04 \pm 7.30)(P<0.05)$ (Table 3$)$.

\section{Association between the K-L grade and the WORMS}

The results of the correlation analyses are shown in Fig. 3. The K-L grade was positively correlated with the patient's age $(\mathrm{Rs}=0.72, P<0.01)$; however, no significant association between the $\mathrm{K}-\mathrm{L}$ grade and gender or side was observed $(P>0.05)$.

The WORMS features were all positively correlated with the K-L grade. The osteophyte score significantly correlated with the K-L grade with an Rs of $0.89(P<0.05)$. The cartilage lesion score and the synovitis score with the SPGR sequence showed moderate correlation with the $\mathrm{K}-\mathrm{L}$ grade with Rs values of 0.66 and 0.41 , respectively $(P<0.05)$. A weak correlation was found between the $\mathrm{K}-\mathrm{L}$ grade and bone marrow abnormalities, bone cysts and joint effusion scores with Rs values of $0.22,0.33$ and 0.38 , respectively $(P<0.05)$.

\section{Association of cartilage lesions with other WORMS features}

The severity of structural joint degeneration was expressed as the sum of WORMS with the SPGR sequence and ranged from 0 to 32 (mean of $9.50 \pm 8.11$ ). The cartilage lesion scores with the SPGR sequence ranged from 0 to 20 (mean of $3.85 \pm 4.72$ ). A weak correlation ( $\mathrm{Rs}=0.24, P<0.05)$ was found between cartilage lesions and bone marrow abnormalities (Fig. 4). In 52 cases of articular cartilage lesions, the prevalence of subchondral bone marrow abnormalities was $71.15 \%$. Of the 32 cases with normal articular cartilage, subchondral bone marrow abnormalities occurred in $40.63 \%$ of patients. A stronger moderate positive correlation was found between osteophytes and cartilage lesion scores (Rs $=0.70, P<0.05)$. The analysis also showed that the cartilage lesion score $(\mathrm{Rs}=0.47, P<0.05)$ and the joint effusion score ( $\mathrm{Rs}=0.44, P<0.05)$ were moderately correlated with synovitis. The bone cyst score weakly correlated with the cartilage lesion score $(\mathrm{Rs}=0.26, P<0.05)$ (Fig. 4).

\section{Discussion}

Currently, in the early evaluation of knee cartilage degeneration, conventional MRI sequences can display the structure of the cartilage and subchondral bone but cannot detect abnormalities in cartilage layers during the early stage of OA [1].

Some studies indicate that in early $\mathrm{OA}$, the moisture and proteoglycan content are altered, and collagen fibres begin to slightly wear and crack on the cartilage surface $[10,11]$. These alterations do not cause changes in the contour of the articular cartilage; therefore, conventional MRI sequences cannot detect this abnormality. Zhao et al. 


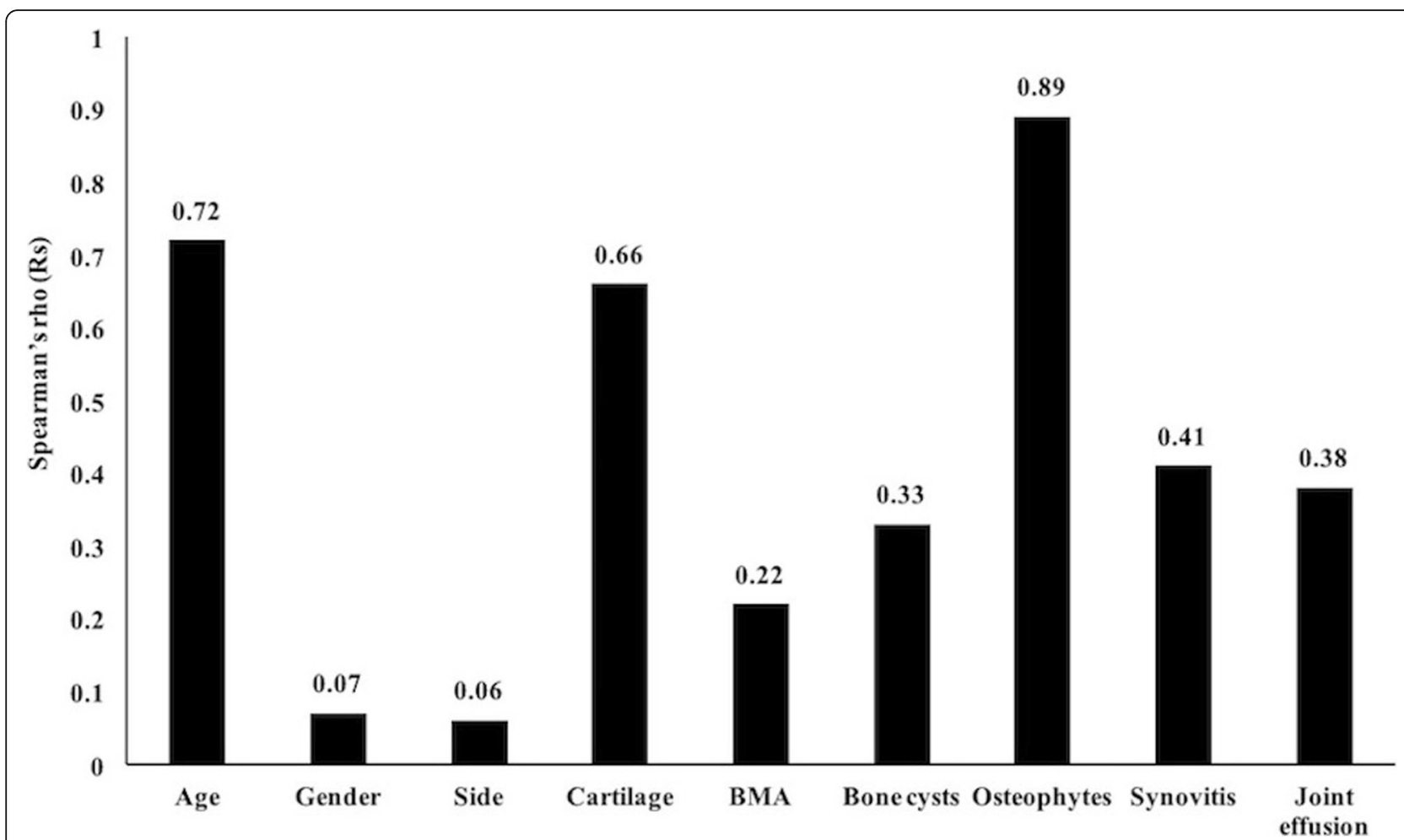

Fig. 3 Correlation between $\mathrm{K}-\mathrm{L}$ and WORMS. The cartilage lesions, osteophytes and synovitis showed moderate positive correlations with $\mathrm{K}$ - $\mathrm{L}$, and weak positive correlation was observed between $\mathrm{K}-\mathrm{L}$ and each of the bone marrow abnormalities, bone cysts and joint effusion. BMA bone marrow abnormalities

[12] examined articular cartilage using the 3D-FS-SPGR sequence and found sensitivity, specificity and Kappa values of $93.1 \%, 98.3 \%$ and 0.849 , respectively.

The main histological characteristic of articular cartilage is its layered structure [13], which includes four layers: the most superficial layer, the transitional layer, the radiation layer and the deepest calcification layer [14]. One study has shown that articular cartilage can present a "high-low-high signal" three-layered structure in the MRI, which is matched by histological stratification [15]. In our study, the use of MRI with the SPGR sequence showed that the normal articular cartilage was represented as a smooth circular arc band with a high signal. The obvious "high-low-high signal" three-layered structure could be detected, and even the five-layered structure could be detected. We considered the three high-signal layers to be the most superficial layer, the transitional layer and the radiation layer, and the low signal layers to be the interfaces between the two layers because of changes in the water and collagen content and tissue anisotropy [15]. The calcification layer was calcified under the tidemark. A previous study reported that the disappearance of the layered collagen structure was the earliest visible morphological change that occurs with OA [16]. Thus, we focused on low K-L grade patients to evaluate the effect of the SPGR sequence on the WORMS. We found that the differences in the cartilage scores and WORMS between the SPGR and $\mathrm{T} 2$ sequences were significant. The frequency of cartilage lesions evaluated by MRI with the SPGR sequence for both K-L1 and K-L2 grades was higher than that evaluated by MRI with the T2 sequence, and the differences in the mean scores for the cartilage lesions between the methods were significant. Even for the K-L3 grade, although the frequency of cartilage lesions was the same between the two approaches, the scores were significantly different. The results of our study demonstrate that the SPGR sequence is more sensitive than the T2 sequence for WORMS evaluation of early cartilage lesions and is advantageous for early detection of OA.

In our study, we found that the WORMS values were generally low with a mean score for cartilage lesions of only $3.85 \pm 4.72$. We speculate that the low $\mathrm{K}$ - $\mathrm{L}$ grade is mainly due to the age distribution of the patients, many of whom were young, which resulted in few patients who had moderate or severe OA or degeneration of the knee joints. However, most of the OA patients were found to have an initial onset in the patellofemoral joint, according to the WORMS evaluation. Cartilage lesions, bone marrow abnormalities and cysts exhibited the highest frequency in the patellofemoral joint, whereas the 


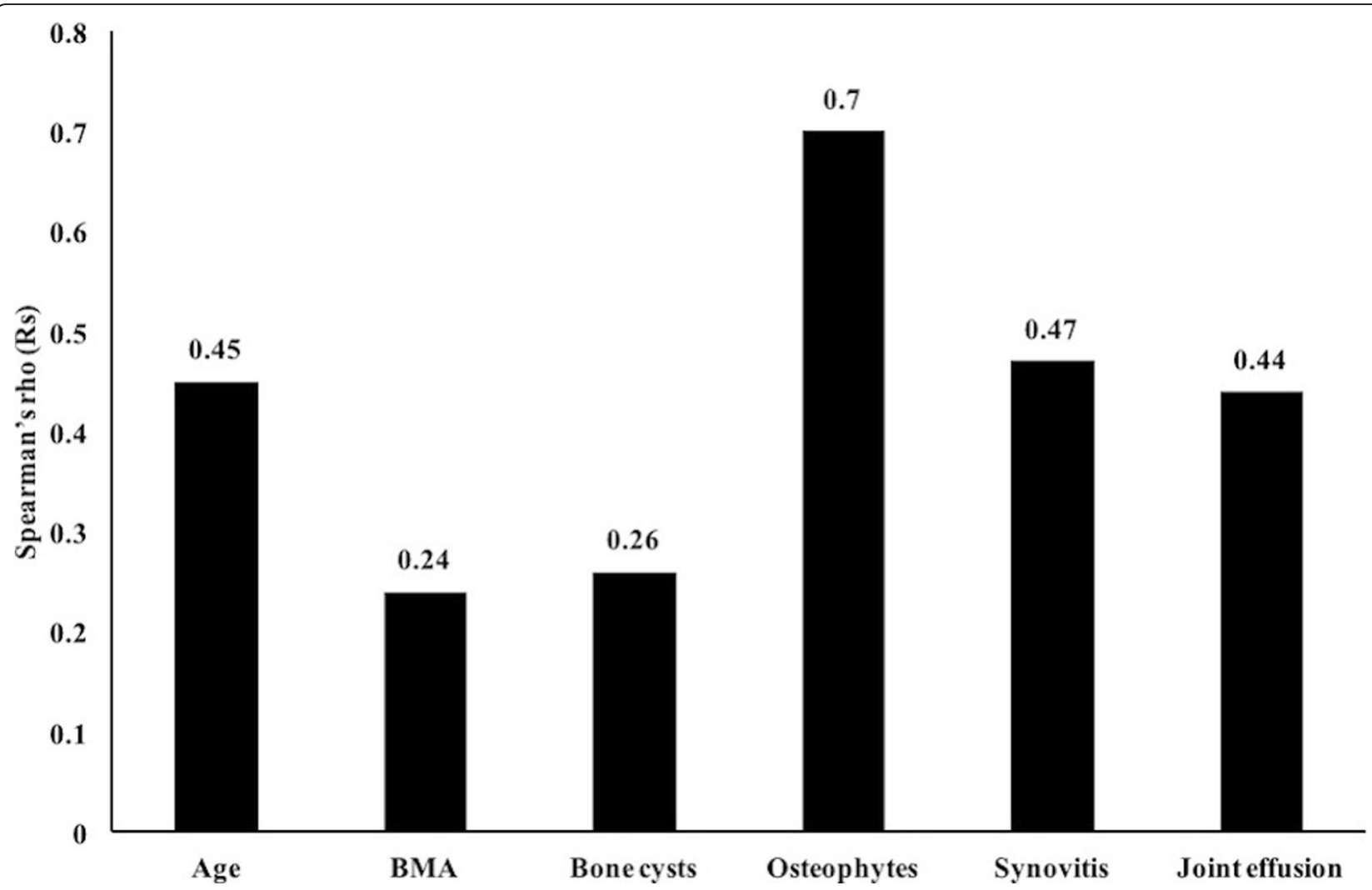

Fig. 4 Correlation between cartilage lesions and other features. Moderate positive correlations were found between the cartilage lesions and each of the following: joint effusion, synovitis, osteophytes, bone cysts, bone marrow abnormalities and age. BMA bone marrow abnormalities

lowest frequency was observed in the intercondylar region. These results are consistent with previous literature [17] and demonstrate the accuracy of the WORMS system. Positive correlations were observed between the $\mathrm{K}-\mathrm{L}$ grade and the features of the modified WORMS, which showed that MRI with the SPGR sequence is more comprehensive and effective than X-rays and MRI with the typically used normal sequence for the early evaluation of OA.

Many studies have observed an association between articular cartilage and subchondral bone and have demonstrated that the mechanical characteristics of subchondral bone and articular cartilage are related to the progression of OA [18-21]. These findings are consistent with the results of our study. We found that in the 52 patients who had mild cartilage lesions, the frequency of bone marrow abnormalities reached $71.15 \%$, which indicated that these articular cartilage lesions were associated with bone marrow abnormalities. The correlation analysis between the features of the modified WORMS showed a positive correlation between cartilage lesions and bone marrow abnormalities. Therefore, we suggest that if conventional MRI sequences show signal changes in the subchondral bone marrow, cartilage lesions should be evaluated with the SPGR sequence.
To confirm negative physical examination of meniscus tears, MRI had been proved to lead to a 2.08 and 2.26 higher rate than patient history and physical examination alone and lead to more than a 10 -fold lower rate of unnecessary surgeries than any other strategy to confirm positive physical examination [22]. Compared to the arthroscopy, MRI was suggested for the diagnosis and therapy of meniscal lesions in the economic and damage consideration [23, 24]. Alizai et al. [25, 26] suggested to use MRI-based semiquantitative grading methods to describe cartilage assessment and provide an update on the risk factors for cartilage loss in knee osteoarthritis. So the modified WORMS evaluation using MRI with the SPGR sequence is suitable for the evaluation of early knee arthritis and will have a bright future with the development of MRI technology.

\section{Conclusions}

We used MRI with the SPGR sequence to determine the modified WORMS, which was better than that found with the conventional sequence for early OA. The SPGR sequence likely increases the sensitivity of the WORMS. Therefore, the SPGR sequence could be used for the evaluation of early knee arthritis in the clinical setting. 


\section{Abbreviations}

3FS-SPGR: Three-dimensional fat-suppressed spoiled gradient-recalled; BMA: Bone marrow abnormalities; CNR: Contrast/noise ratio; K-L: KellgrenLawrence; LFTJ: Lateral tibiofemoral joint; MFTJ: Medial tibiofemoral joint: MRI: Magnetic resonance imaging; OA: Osteoarthritis; PFJ: Patellofemoral joint; Rs: Spearman's rho; S: Subspinous; WORMS: Whole-Organ Magnetic Resonance Imaging Score

\section{Acknowledgements}

Not applicable

\section{Funding}

This research was supported by the China Postdoctoral Science Foundation (2018 M642459).

\section{Availability of data and materials}

The datasets used and/or analysed during the current study are available from the corresponding author on reasonable request.

\section{Authors' contributions}

$X Y, Z Y L$ and YPC contributed to the conception and design of the experiment. $X Y, Z Y L, Y F X, H Y$ and $X L$ gathered all data and verified the analytical data. ZCM, HL and RW contributed to the statistical analysis and helped interpret the results. XY and YPC supervised the discussion with ZYL. YFX and HW wrote the manuscript. All authors discussed the final results and approved the final manuscript.

\section{Ethics approval and consent to participate}

This study was approved by the Ethics Committee of Peking University First Hospital. All participants received informed consent.

\section{Competing interests}

The authors declare that they have no competing interests.

\section{Publisher's Note}

Springer Nature remains neutral with regard to jurisdictional claims in published maps and institutional affiliations.

\section{Author details}

'Department of Orthopedics, Peking University First Hospital, No. 8, Xishiku Street, Beijing 100034, Beijing, China. ${ }^{2}$ Department of Orthopedics, Zhejiang University School of Medicine First Affiliated Hospital, No. 79, Qingchun Road, Hangzhou 310000, Zhejiang, China. ${ }^{3}$ Department of Radiology, Peking University First Hospital, No. 8, Xishiku Street, Beijing 100034, Beijing, China.

Received: 15 January 2019 Accepted: 26 April 2019

Published online: 24 May 2019

\section{References}

1. Hodler J, Berthiaume MJ, Schweitzer ME, Resnick D. Knee joint hyaline cartilage defects: a comparative study of MR and anatomic sections. J Comput Assist Tomoger. 1992;16:597-603.

2. Blankenbaker DG, Ullrick SR, Kijowski R, Davis KW, De Smet AA, Shinki K, et al. MR arthrography of the hip: comparison of IDEAL-SPGR volume sequence to standard MR sequences in the detection and grading of cartilage lesions. Radiology. 2011;261:863-71.

3. Hochberg MC, Altman RD, Brandt KD, Clark BM, Dieppe PA, Griffin MR, et al. Guidelines for the medical management of osteoarthritis, part II (osteoarthritis of the knee). Arthritis Rheum. 1995:38:1541-6.

4. Woo A, Lechner B, Fu T, Wong CS, Chiu N, Lam H, et al. Cut points for mild, moderate, and severe pain among cancer and non-cancer patients: a literature review. Ann Palliat Med. 2015;4:176-83.

5. Reddi D, Curran N. Chronic pain after surgery: pathophysiology, risk factors and prevention. Postgrad Med J. 2014:90:222-7.

6. Kellgren JH, Lawrence JS. Radiological assessment of osteoarthritis. Ann Rheum Dis. 1957:16:494-502

7. Peterfy CG, Guermazi A, Zaim S, Tirman PF, Miaux Y, White D, et al. WholeOrgan Magnetic Resonance Imaging Score (WORMS) of the knee in osteoarthritis. Osteoarthritis Cartilage. 2004;12:177-90.
8. Recht MP, Kramer J, Marcelis S, Pathria MN, Trudell D, Haghighi P, et al. Abnormalities of articular cartilage in the knee: analysis of available MR techniques. Radiology. 1993;187:473-8.

9. Cohen J. Statistical power analysis for the behavioral sciences. 2nd ed. Hillsdale: L.Erlbaum Associates; 1988.

10. Madry H, Kon E, Condello V, Peretti GM, Steinwachs M, Seil R, et al. Early osteoarthritis of the knee. Knee Surg Sports Traumatol Arthrosc. 2016;24(6): 1753-62.

11. Kim JR, Yoo JJ, Kim HA. Therapeutics in osteoarthritis based on an understanding of its molecular pathogenesis. Int J Mol Sci. 2018;19(3).

12. Zhao G, Liu Y, Qu F, Yuan B, Wang J, Shen X, et al. The value of 3 dimensional-fat suppression-spoiled gradient-recalled acquisition sequence on single compartment osteoarthritis for unicompartmental arthroplasty preoperative assessment. Zhonghua Wai Ke Za Zhi. 2015;53:528-32.

13. Fujioka R, Aoyama T, Takakuwa $T$. The layered structure of the articular surface. Osteoarthritis Cartilage. 2013;21(8):1092-8.

14. Chen T, Bai J, Tian J, Huang P, Zheng H, Wang J. A single integrated osteochondral in situ composite scaffold with a multi-layered functional structure. Colloids Surf B Biointerfaces. 2018;167:354-63.

15. Setton LA, Elliott DM, Mow VC. Altered mechanics of cartilage with osteoarthritis: human osteoarthritis and an experimental model of joint degeneration. Osteoarthritis Cartilage. 1999;7:2-14.

16. Mosher TJ, Dardzinski BJ. Cartilage MRI T2 relaxation time mapping: overview and applications. Semin Musculoskelet Radiol. 2004;8:355-68.

17. Sharma L, Chmiel JS, Almagor O, Dunlop D, Guermazi A, Bathon JM, et al. Significance of preradiographic magnetic resonance imaging lesions in persons at increased risk of knee osteoarthritis. Arthritis Rheumatol. 2014; 66(7):1811-9.

18. Goldring SR, Goldring MB. Changes in the osteochondral unit during osteoarthritis: structure, function and cartilage-bone crosstalk. Nat Rev Rheumatol. 2016;12(11):632-44.

19. Adebayo OO, Ko FC, Wan PT, Goldring SR, Goldring MB, Wright TM, et al. Role of subchondral bone properties and changes in development of loadinduced osteoarthritis in mice. Osteoarthritis Cartilage. 2017;25(12):2108-18.

20. Aho OM, Finnilä M, Thevenot J, Saarakkala S, Lehenkari P, et al. Subchondral bone histology and grading in osteoarthritis. PLoS One. 2017;12(3): e0173726.

21. Finnilä MAJ, Thevenot J, Aho OM, Tiitu V, Rautiainen J, Kauppinen S, et al. Association between subchondral bone structure and osteoarthritis histopathological grade. J Orthop Res. 2017;35(4):785-92.

22. Mather RC 3rd, Garrett WE, Cole BJ, Hussey K, Bolognesi MP, Lassiter T, et al. Cost-effectiveness analysis of the diagnosis of meniscus tears. Am J Sports Med. 2015;43(1):128-37.

23. Weinstabl $R$, Muellner $T$, Vécsei $V$, Kainberger $F$, Kramer M. Economic considerations for the diagnosis and therapy of meniscal lesions: can magnetic resonance imaging help reduce the expense? World J Surg. 1997; 21(4):363-8

24. Ryzewicz M, Peterson B, Siparsky PN, Bartz RL. The diagnosis of meniscus tears: the role of MRI and clinical examination. Clin Orthop Relat Res. 2007; 455:123-33

25. Alizai H, Virayavanich W, Joseph GB, Nardo L, Liu F, Liebl H, et al. Cartilage lesion score: comparison of a quantitative assessment score with established semiquantitative MR scoring systems. Radiology. 2014;271(2):479-87.

26. Alizai H, Roemer FW, Hayashi D, Crema MD, Felson DT, Guermazi A. An update on risk factors for cartilage loss in knee osteoarthritis assessed using MRI-based semiquantitative grading methods. Eur Radiol. 2015;25(3):883-93.

Ready to submit your research? Choose BMC and benefit from

- fast, convenient online submission

- thorough peer review by experienced researchers in your field

- rapid publication on acceptance

- support for research data, including large and complex data types

- gold Open Access which fosters wider collaboration and increased citations

- maximum visibility for your research: over $100 \mathrm{M}$ website views per year

At BMC, research is always in progress.

Learn more biomedcentral.com/submissions 